\title{
La inclusión educativa en la enseñanza superior: retos y demandas
}

\section{Educação inclusiva e ensino superior: desafios e demandas}

\section{Educational inclusion in higher education: challenges and demands}

\author{
Maria José Bagnato*
}

\begin{abstract}
RESUMEN
Desde la perspectiva de derechos no cabe duda sobre la necesidad imperiosa de habilitar los espacios de inclusión educativa en la enseñanza superior, en particular para las personas con discapacidad. Sin embargo, el ejercicio de derechos, en este caso, requiere ciertas modificaciones políticas, estructurales y procedimentales que incluyen un cambio de perspectiva cultural. Para ello, este artículo se propone como objetivo, a partir de los marcos conceptuales que se plantean tanto en la Convención sobre los Derechos de las Personas con Discapacidad como en la Clasificación Internacional sobre el Funcionamiento, la Discapacidad y la Salud, reflexionar sobre su aplicabilidad en el acceso a la educación terciaria. Asimismo, y como consecuencia, establecer las posibles contradicciones que se plantean a la luz de los prejuicios que subsisten hacia las personas con discapacidad, a la hora de ejercer distintos roles profesionales. Se trata, por lo tanto, de un artículo teórico, basado en el análisis documental internacional y artículos científicos, tomando como estudio de caso la situación de Uruguay. Se pretende, como resultado, aportar al debate actual sobre esta temática, desde un punto de vista teórico, técnico y operativo.
\end{abstract}

Palabras claves: Inclusión. Educación terciaria. Discapacidad.

* Universidad de la Republica del Uruguay (Udelar). Facultad de Psicología. Montevideo, Uruguay. Calle Tristán Navaja, nº 1674. Edificio Central. E-mail: majose@psico.edu.uy 


\title{
RESUMO
}

Do ponto de vista dos direitos humanos, não há dúvida da necessidade imperativa de proporcionar espaços de educação inclusiva no ensino superior, em particular para pessoas com deficiência. No entanto, a prática dos direitos, nesse caso, exige mudanças políticas, estruturais e processuais, que incluem transformações nas perspectivas culturas. Nesse sentido, este artigo tem como objetivo refletir sobre a aplicabilidade dos quadros da Convenção sobre os Direitos das Pessoas com Deficiência e da Classificação Internacional de Funcionamento, Incapacidade e Saúde no acesso ao ensino superior. Além disso, como consequência, visa identificar possíveis contradições que surgem à luz do preconceito que ainda se têm em relação às pessoas com deficiência ao desempenhar diferentes papéis profissionais. Portanto, trata-se de um artigo teórico, baseado em análise de documentos internacionais e artigos científicos, tomando como estudo de caso a situação do Uruguai. Pretende-se como resultado, contribuir para o debate atual sobre o tema a partir de um ponto de vista teórico, técnico e operacional.

Palavras-chave: Inclusão. Ensino superior. Incapacidade.

\begin{abstract}
From a Human Rights perspective, there is no doubt in the imperative need to provide spaces of educational inclusion in higher education, in particular for persons with disabilities. However, the practice of rights, in this case, requires political, structural and procedural changes, that includes transformations in cultural perspectives. This article aims to reflect on the applicability of the frameworks of Convention on the Rights of Persons with Disabilities and the International Classification of Functioning, Disability and Health, in the access to tertiary education. Thus, and as a result, it aims to identify potential contradictions that arise in the light of the prejudice that people still have towards persons with disabilities, when performing several professional roles. Therefore, it is a theoretical article based on documental analysis, which includes international documents, scientific papers, and considers the situation of Uruguay as a case study. The intended outcome of this article is to make a contribution to the current debate on the topic from a theoretical, technical and operational approach.
\end{abstract}

Keywords: Inclusion. Tertiary education. Disability. 


\section{Educación y discapacidad}

Los países que han ratificado el protocolo facultativo de la Convención sobre los Derechos de las Personas con Discapacidad (ONU, 2006) tienen el mandato de armonizar todos los marcos legales, las prácticas y los dispositivos para garantizar sus derechos. Sin embargo, la existencia de la ley no conlleva necesariamente la toma de medidas, si no se establecen mecanismos de control. Aun así, la aplicación de la convención en todos sus términos requiere un profundo proceso de análisis y conceptualización sobre las prácticas, para que las mismas sean sostenibles.

Cuando hablamos de inclusión educativa en la enseñanza superior, estamos hablando del nivel más alto en la cadena educativa formal. Por lo tanto, el acceso a este nivel va a estar condicionado por el acceso educativo a los niveles anteriores. De acuerdo con el Informe Mundial sobre la Discapacidad (OMS, 2011b), históricamente, tanto niños como adultos con discapacidad han sido excluidos de la educación formal. Según dicho informe, entre los 51 países incluidos en el estudio, el 50,6\% de los hombres con discapacidad terminaron la escuela primaria, en comparación con el $61,3 \%$ de los hombres sin discapacidad. El 41,7\% de las mujeres con discapacidad terminaron la escuela primaria en comparación con el $52,9 \%$ de las mujeres sin discapacidad. No sorprende, entonces, una menor presencia de estudiantes con discapacidad en los niveles superiores.

Si tomamos en cuenta las definiciones actuales acordadas por los principales organismos internacionales, tanto Naciones Unidas (ONU, 2006) como la Organización Mundial de la Salud (OMS, 2011a), observamos que, a pesar de surgir de orígenes distintos, ambas definiciones coinciden en tres aspectos. Por orígenes distintos, nos referimos al modelo explicativo social en el primer caso y al modelo bio-psico-social, en el segundo caso.

El primer aspecto fundamental es que la situación de discapacidad siempre tiene su origen en la condición de salud de una persona, esto es, la presencia de deficiencias físicas, mentales, intelectuales o sensoriales en tiempo prolongado. Por lo tanto, no podemos hablar de discapacidad en sentido abstracto y sin la constatación de la condición de salud como deficiencia.

El segundo aspecto fundamental es que la discapacidad, como tal, no es una situación que "porte" la persona, sino que se produce en interacción con su entorno o ambiente. De forma que, entre más grandes sean las barreras, más incrementaran los niveles de discapacidad. Es decir que se traslada la perspectiva de la discapacidad del nivel personal al social. Lo que implica, claramente, 
que la deficiencia por sí misma no constituye discapacidad, en su terminología actual, y por lo tanto queda claro que discapacidad no es sinónimo de patología.

El tercer aspecto fundamental es que tanto la CDPD como la CIF son acuerdos internacionales generales, que cada país debe adaptar a su contexto. De manera que las recomendaciones no pretenden privilegiar a las personas con discapacidad del resto de la población, sino por el contrario, garantizarles las oportunidades para el ejercicio de los derechos en igualdad de condiciones que la población general. Las medidas de discriminación positiva, en ningún caso, pueden entenderse como beneficios, sino como equiparación para el ejercicio de derechos y responsabilidades.

Si bien estas posturas conllevan cambios de enfoque y con ello cambio en las prácticas profesionales hacia la discapacidad, es muy difícil desprender de las representaciones sociales la idea de que la discapacidad es una situación personal y asociada a la deficiencia. Esto se profundiza cuando la persona, además, se encuentra en una situación de alta dependencia, entendiendo dependencia como cuando alguien que requiere a otra persona para el desarrollo de las actividades básicas de la vida, tales como alimentación, vestido, cuidado propio o participar por sí misma en actividades de estudio, recreación o trabajo. Es por ello que, en el presente artículo, intentaremos dar un punto de vista que aporte a la reflexión de las posibilidades de aplicación de los modelos explicativos basados en la relación persona/contexto, específicamente en el ámbito de la educación superior.

\section{Niveles de interacción para la aplicación de la inclusión}

Proponemos que deben pensarse las posibilidades de aplicación de la inclusión en tres niveles diferenciados pero articulados entre sí:

- Un nivel político, es decir, de toma de decisiones que explícitamente propongan la inclusión y promuevan acciones que la viabilicen con adjudicación de recursos para ello.

- Un nivel estructural, aquello que implica la materialidad de los acontecimientos. Donde incluiríamos los conceptos de eliminación de barreras físicas y comunicacionales, al amparo de la accesibilidad universal.

- Un nivel procedimental, el cual apunta a la eliminación de barreras actitudinales, que además requieren establecimientos de protocolos, posibilidades de ajustes razonables, flexibilidad y adecuación curricular.

Integralmente, estos niveles apuntan a un cambio sociocultural que impactaría positivamente tanto en el interior de las Universidades o entidades de Educación Superior, como en la sociedad en su conjunto. 
Hablar de inclusión educativa a nivel superior implica hablar del acceso, pero también de la permanencia y del egreso. Esto conlleva diferentes connotaciones, cuando hablamos de eliminación de barreras arquitectónicas, en la comunicación y actitudinales, para que las personas puedan circular en el contexto edilicio de las universidades. Sin embargo, la permanencia implica ir más allá, abarcando lo que se denominan adaptaciones curriculares. Estas adaptaciones, muchas veces, pueden implicar la necesidad, no de adaptar los objetivos de la formación, sino los modos de transitarla, dependiendo del tipo de requerimientos de las personas en situación de discapacidad, modificaciones en la recepción del material de estudio, en las posibilidades de prácticas, en las pruebas de rendimiento y en las metodologías. Estaríamos hablando de lo que puede denominarse flexibilidad curricular, que no implica, en ningún caso, reducir la calidad de la formación. A su vez, el egreso nos lleva a tomar en cuenta los prejuicios que a nivel social aparecen hacia las personas en situación de discapacidad, cuando puede cuestionarse el ejercicio de determinadas profesiones.

Los modelos explicativos hacia la discapacidad que han existido históricamente se plantean como excluyentes entre sí. Pues, por ejemplo, el modelo social de la discapacidad aparece como antagonista al modelo rehabilitador, ya que el último se centra en la persona y el primero se centra en las barreras del entorno. Sin embargo, hoy podemos observar que si tomamos en cuenta la definición de los organismos internacionales la CDPC (ONU, 2006) como la Clasificación Internacional del Funcionamiento de la Discapacidad y de la Salud (OMS, 2011a), ambas reconocen la necesidad de identificar las limitaciones particulares para poder identificar tanto las barreras como los facilitadores. De eso se trata cuando se habla de ajustes razonables (ONU, 2006, art. $2^{\circ}$ ).

Por ende, tal como se plantea, la discapacidad no es un concepto absoluto, sino relativo. Una persona puede tener deficiencias, aún en sus grados de mayor severidad, incluso con altos niveles de dependencia; pero la discapacidad va a estar presente si esa persona no accede a las mismas posibilidades que tiene el resto de la población, es decir, de acceso a bienes y servicios que le permitan la plena inclusión y participación social.

Esta situación nos lleva a un falso antagonismo y cuestionar si determinar la discapacidad de una persona, es necesaria para desempeñarse en los distintos ámbitos de la vida. En particular, si nos centramos en la educación superior, podríamos decir que no sería necesario centrarse en la persona, sino en la eliminación de barreras. En consecuencia, la concepción de accesibilidad universal y de ajustes razonables son herramientas para ello, sustituyendo, así, la noción de necesidades educativas especiales (NEE). El concepto de NEE (ESPAÑA, 1994) ha sido un avance en materia de inclusión educativa, sin embargo, podemos decir que el estudiante tal vez no presente NEE, sino que lo que requiere son ajustes 
razonables para desarrollar su proceso de enseñanza-aprendizaje. Hablar de ajustes razonables, combinando la accesibilidad universal, implica adecuar los espacios físicos de forma que sean disponibles para todas las personas en relación a sus necesidades de desplazamiento y uso de las instalaciones, la circulación lo más autónoma posible, más otorgar un sistema de apoyos específicos para aquellas personas que lo requieran. Allí, entonces, nos encontramos con los niveles planteados: tanto el estructural como la adecuación de entornos arquitectónicos y comunicacionales; y el procedimental, que requiere de las actitudes positivas de los funcionarios docentes y no docentes, para el establecimiento de procedimientos protocolizados, donde incluiríamos la adaptación curricular y los apoyos técnicos y tecnológicos como parte de los ajustes razonables. Se entiende por adaptación curricular toda acción que el docente facilite el ajuste en adecuación metodológica, de actividades, de evaluación y accesibilidad para todos los contenidos (LUQUE-PARRA; RODRÍGUEZ-INFANTE; LUQUE-ROSAS, 2016).

Un debate importante se produce cuando se considera si los profesores y maestros deben tener una formación específica para ejercer su rol para, de esta manera, incluir o si es suficiente ser flexibles a la hora de atender estos ajustes. Para la educación superior parece más evidente que el último enfoque sea el más razonable. Primero, porque los estudiantes que acceden al nivel superior ya han incorporado los conocimientos y estrategias requeridas para ingresar a la Universidad. Al igual que cualquier estudiante, el requisito es haber aprobado el nivel secundario. Segundo, es la propia persona que conoce sus requerimientos de apoyo. En este sentido, la persona en situación de discapacidad es quien puede orientar sobre los recursos necesarios y, en este caso, además de la participación del docente que adapte su modalidad o sus requisitos, la propia persona debería ser un participante activo en su proceso formativo, al igual que otros profesionales. Como tales nos referimos, por ejemplo, a la figura del asistente personal o al personal de apoyo para recursos informáticos, o de comunicación, como podría ser el caso de intérpretes en lengua de señas, personal para la transcripción de textos al braille, entre otros que dan soporte a la formación. Tan relevante como los apoyos humanos son las tecnologías de asistencia y las ayudas técnicas que emplea la persona en su vida cotidiana y es indispensable dentro de los establecimientos educativos (GÓMEZ-BELEÑO; LÓPEZ-MUÑOZ, 2016).

En general, como es el caso de Uruguay, vemos que las experiencias de inclusión en educación superior son experiencias puntuales a partir del tránsito de sujetos particulares, por lo que muchas veces conlleva a un esfuerzo personal que debe ir abriendo camino para poder transitar en su propia formación. Como resulta de las recomendaciones de la CDPD, no se puede garantizar la accesibilidad universal en la educación superior si no se establecen políticas claras, 
tal como planteáramos. Esto representa uno de los niveles fundamentales que pueden ser adoptados por todos los Servicios y por las diferentes formaciones en carreras de grado y de posgrados. Entre otros aspectos es importante contar con una reglamentación clara que posibilite el financiamiento y establezca mecanismos para el acceso a los apoyos requeridos (TENORIO EITEL; RAMÍREZ-BURGOS, 2016).

\section{El caso de Uruguay}

Uruguay cuenta con una única universidad pública y gratuita, la Universidad de la República (UdelaR). De acuerdo con el VII Censo de Estudiantes de Grado (UDELAR, 2012), en la UdelaR hay 85.905 estudiantes activos, de los cuales, el 1,3\% se ha identificado con alguna discapacidad total o parcial. Cabe señalar que las respuestas habitualmente remiten a las deficiencias, por lo que aparecen reconocidas las deficiencias referidas a las situaciones de discapacidad auditivas (sordera e hipoacusia), visuales (ceguera y baja visión) y motrices. Un dato importante por considerar es que el porcentaje es menor cuando se trata de responder si la discapacidad es total, frente a las respuestas de discapacidad parcial. Asimismo, se constata que la Universidad, a través de sus diferentes servicios, ha avanzado en materia de accesibilidad, aunque no de forma planificada estratégicamente. Presentamos en la Tabla 1, la descripción de los avances en materia de inclusión de personas con discapacidad.

Como puede apreciarse en la Tabla 1, se ha promovido un avance en las condiciones de acceso para la inclusión; sin embargo, este avance ha dependido de las personas que han logrado acceder a la universidad apreciándose fundamentalmente modificaciones a nivel estructural (supresión de barreras arquitectónicas), siendo la excepción, a la fecha del relevamiento de información, la facultad de Ciencias Sociales, donde se plantean aspectos procedimentales a través de la atención y apoyo a los estudiantes, aunque no se mencionan protocolos y otras situaciones de sensibilización, pero no continuas.

Por otra parte, pueden identificarse como políticas a nivel central de la UdelaR:

- Portal web: a partir de las pautas de accesibilidad contenido Web 2.0 establecidas por el consorcio mundial www se garantiza que los contenidos visuales estén disponibles en texto y los contenidos de texto disponibles en audio. Se incorpora la posibilidad de acceder por teclado a los contenidos del portal. Chequeo entre los enlaces y su correcta descripción, jerarquización de los 
TABLA 1 -AVANCES EN ACCESIBILIDAD POR FACULTADES DE LA UDELAR

\begin{tabular}{|c|c|c|}
\hline Facultad & Accesibilidad & Descripción \\
\hline $\begin{array}{l}\text { Derecho. Edificio central } \\
\text { de la Udelar }\end{array}$ & Accesibilidad Física & $\begin{array}{l}\text { Construcción de un sistema de tres rampas, } \\
\text { colocación de pasamanos y plataforma sal- } \\
\text { va-escaleras en acceso principal. }\end{array}$ \\
\hline $\begin{array}{l}\text { Centro de Diseño. } \\
\text { Facultad de Arquitectura }\end{array}$ & Accesibilidad Física & $\begin{array}{l}\text { Construcción de rampa de acceso al Edi- } \\
\text { ficio Central. }\end{array}$ \\
\hline Centro de Diseño. & Sensibilización & $\begin{array}{l}\text { Realización de jornadas de sensibili- } \\
\text { zación Proyectos estudiantiles sobre } \\
\text { "Indumentaria y Discapacidad". }\end{array}$ \\
\hline $\begin{array}{l}\text { Facultad de Humanidades } \\
\text { y Ciencias de la Educación }\end{array}$ & $\begin{array}{l}\text { Accesibilidad en la } \\
\text { Información y } \\
\text { Comunicación. }\end{array}$ & $\begin{array}{l}\text { Permite al usuario ajustar las característi- } \\
\text { cas del sitio a sus necesidades visuales y } \\
\text { auditivas, estando el equipo técnico traba- } \\
\text { jando para su pronta migración a una nue- } \\
\text { va plataforma que mejore las aplicaciones } \\
\text { directamente vinculadas a la accesibilidad } \\
\text { del mismo. }\end{array}$ \\
\hline $\begin{array}{l}\text { Facultad de Humanidades } \\
\text { y Ciencias de la Educación }\end{array}$ & Sensibilización & $\begin{array}{l}\text { Realización de jornadas de sensibilización } \\
\text { de la LSU en Facultad, abiertas a todo } \\
\text { público. }\end{array}$ \\
\hline $\begin{array}{l}\text { Facultad de Psicología } \\
\text { - Universidad de la } \\
\text { República }\end{array}$ & $\begin{array}{l}\text { Accesibilidad al medio } \\
\text { físico }\end{array}$ & $\begin{array}{l}\text { Diseño de un sistema de puentes metálicos } \\
\text { que permite el acceso a la totalidad de los } \\
\text { niveles del edificio y a todas sus instala- } \\
\text { ciones: Institutos, oficinas administrativas, } \\
\text { aulas, laboratorios y espacios de cogobier- } \\
\text { no. Colocación de ascensor que permite el } \\
\text { acceso a todos los niveles. } \\
\text { Señalización y rampas exteriores para el } \\
\text { ingreso al edificio. Reserva de estaciona- } \\
\text { miento para vehículo adaptado. } \\
\text { Diseño de baños inclusivos. }\end{array}$ \\
\hline $\begin{array}{l}\text { Facultad de Ciencias } \\
\text { Sociales }\end{array}$ & $\begin{array}{l}\text { Atención y seguimiento para } \\
\text { la permanencia y egreso exi- } \\
\text { toso de las personas en si- } \\
\text { tuación de discapacidad que } \\
\text { concurren a esta casa de es- } \\
\text { tudios, mediante la creación } \\
\text { de un cargo de ayudante para } \\
\text { el cumplimiento de funciones } \\
\text { de Información y Orientación } \\
\text { a Estudiantes sobre el Plan } \\
\text { de Estudios, específicamente } \\
\text { orientado hacia la atención y } \\
\text { seguimiento de los estudiantes } \\
\text { en situación de discapacidad. }\end{array}$ & $\begin{array}{l}\text { Atención de estudiantes en situación de } \\
\text { discapacidad, coordinación con docentes; } \\
\text { coordinación con la administración de la } \\
\text { enseñanza e instituciones de referencia } \\
\text { que contribuyan a potenciar las condicio- } \\
\text { nes de permanencia y egreso exitoso. } \\
\text { Señalizar en la calle (sobre la banquina del } \\
\text { edificio) estacionamiento para vehículos } \\
\text { adaptados. } \\
\text { Acceso a la información para estudiantes } \\
\text { sordos. } \\
\text { Capacitación a funcionarios en lengua de } \\
\text { señas. } \\
\text { Accesibilidad al medio físico. }\end{array}$ \\
\hline $\begin{array}{l}\text { Facultad de Ciencias } \\
\text { Económicas y } \\
\text { Administración }\end{array}$ & $\begin{array}{l}\text { Accesibilidad al medio físico } \\
\text { e identificación de necesi- } \\
\text { dades de estudiantes. }\end{array}$ & $\begin{array}{l}\text { Eliminación de rampa provisoria no accesible } \\
\text { y habilitación de entrada al aulario accesible. } \\
\text { Relevamiento de estudiantes con dis- } \\
\text { capacidad y sus requerimientos. }\end{array}$ \\
\hline
\end{tabular}

FUENTE: elaborado a partir de la información que figura en http://www.accesibilidad.gub.uy/ wp-content/uploads/2013/08/Agenda-2012-Segundo-Semestre.pdf 
contenidos y la incorporación de encabezados, fundamentales para la utilización de software lectores de pantalla.

- Bienestar Estudiantil: programa de apoyo a estudiantes sordos de todas las carreras, mediante el cual se ofrece el servicio de intérpretes de lengua de señas en todas las actividades curriculares que estudiantes requieran.

Otro aspecto que puede verse como oportunidad, en el caso de la UdelaR, es la propuesta de reforma en los planes de estudios con criterio de flexibilidad curricular. Cada vez más, la formación universitaria se plantea con espacios curriculares flexibles para todos los estudiantes. Así, por ejemplo, la acreditación de asignaturas para superar los ciclos formativos puede ser una opción válida que permita al estudiante, que recorre su tránsito curricular, en los ciclos más avanzados, seleccionar aquellas asignaturas que pueden ser de su interés $\mathrm{y}$, a la vez, garantizar sus posibilidades de cursarla. Esto implica, por otra parte, identificar trayectos formativos y, en alguna medida, puede facilitar el perfil de egreso. En Uruguay está garantizada la educación pública y gratuita como derecho, que conlleva a la habilitación profesional, pero también es importante recordar que la producción de conocimiento es una de las líneas prioritarias de las universidades, por lo que también deberíamos incorporar que las personas en situación de discapacidad pueden dedicarse a la investigación en sus distintos campos.

Los docentes universitarios y los funcionarios no docentes no están por fuera de las representaciones sociales y, por lo tanto, una sociedad con mayores prejuicios va a repercutir también en el ámbito universitario. No obstante, entendemos que debería ser una responsabilidad de la educación superior aportar a la transformación de estas representaciones negativas acerca de la discapacidad.

Simultáneamente, tal como lo indica la convención, las formaciones deberían incluir la temática de la discapacidad como parte de los planes de estudio, y esto redundaría en mejorar las concepciones que tienen los profesionales como, por ejemplo, de la salud o de las ciencias tecnológicas y sociales que egresan de las universidades. Se debe tener en cuenta que, muchas veces, los profesionales se desempeñan como asesores políticos y, precisamente, son las políticas y los marcos normativos y legales en el país los que van a favorecer o no los cambios. Debería existir una sinergia entre las posibilidades de acceso de las personas en situación de discapacidad como futuros profesionales y otros universitarios que estén preparados técnica y conceptualmente sobre las nuevas perspectivas que se plantean. Se puede afirmar que todas las áreas de conocimiento son importantes para la inclusión de las personas con discapacidad. Así, las áreas educativas, las áreas sociales, de la salud y científico-tecnológicas deberían ser quienes aporten en el campo social para que sucedan estas transformaciones. 


\section{Consideraciones finales}

Para avanzar en la perspectiva de derechos hacia las personas en situación de discapacidad, no es suficiente contar con leyes que lo amparen. Poner en práctica las recomendaciones de la Convención sobre los Derechos de las Personas con Discapacidad conlleva una reflexión permanente sobre prácticas y dispositivos que viabilicen y le den sostenibilidad al ejercicio de derechos y responsabilidades.

Realizar ajustes razonables, así como tomar medidas de discriminación positiva, no implica en absoluto una actitud asistencialista, sino como planteamos anteriormente, una acción de equiparación de oportunidades. Incluso la experiencia de inclusión de los propios actores involucrados resulta en aportes concretos como antecedentes de experiencias positivas para aportar a la generalización de la modificación en los distintos niveles (YUPANQUI CONCHA; ARANDA FARÍAS; VÁSQUEZ OYARZUN; VERDUGO HUENUMÁN, 2014).

Hemos propuesto tres niveles centrales que pueden orientar las prácticas. Estos son los niveles político, estructural y procedimental, los cuales requieren una interacción permanente para garantizar la coherencia de las mismas. Al igual que en todos los ámbitos de la vida: (a) las definiciones políticas en consonancia con los objetivos de la convención, (b) las modificaciones estructurales que permitan garantizar la accesibilidad universal y (c) los procedimientos y mecanismos para el ajuste persona-medio, en la educación superior, se refleja en las posibilidades de acceso, tránsito y egreso de las personas en situación discapacidad. En el caso de Uruguay, no parecen, en sí mismos, cada uno de los niveles estar vinculados; con todo, se suelen observar medidas parciales en cada uno de ellos. O sea, por ejemplo, definiciones políticas que no conllevan adjudicación de presupuesto, transformaciones edilicias que no contemplan eliminar barreras de la comunicación y modificaciones en los procedimientos que no logran protocolizarse.

Si bien identificamos dos medidas muy importantes desde el centro: accesibilidad al portal web de la UdelaR y el servicio de intérpretes en lengua de señas, el acceso, permanencia y egreso de personas con discapacidad aún no está garantizada. Se puede reconocer un avance en el interés político-académico en sus autoridades centrales, las cuales permitirían iniciar un proceso de mejora. Insistimos, entonces, que la definición de políticas centrales, con la adjudicación de recursos presupuestales es la clave para concretar una UdelaR verdaderamente inclusiva.

Planteábamos, además, que no es favorable el concepto de NEE para el caso de universitarios, ya que podría contribuir a la inseguridad de los docentes 
sobre las propias posibilidades de desplegar los procesos de enseñanza-aprendizaje. Plantear la identificación de necesidad de ajustes razonables coloca a docente-estudiante en un vínculo colaborativo para compartir ese proceso. De forma que son los propios estudiantes en situación de discapacidad que ya han transitado en el proceso educativo de los niveles anteriores quienes están en condiciones de proponer los ajustes.

Por último, importa cuestionarnos acerca de cuál es la responsabilidad de la Universidad frente a los demás sectores de la educación y de qué manera podría contribuir para que las personas puedan equipararse en sus niveles educativos desde el inicio de su educación formal. Si bien sostenemos que conocer las limitaciones de la persona para la educación superior no sería necesario, sí creemos que, cuando la situación de discapacidad se da en edades tempranas de la vida, los profesionales que intervienen en las orientaciones para la escolarización deberían tener claridad sobre sus indicaciones, tanto pedagógicas como de fortalecimiento de sus capacidades. En este caso, la articulación entre los subsistemas salud y educación resulta prioritario y, precisamente, la determinación de necesidades de apoyo requiere de equipos profesionales calificados que puedan actuar en forma interdisciplinaria junto con el maestro o maestra. Este equipo debería facilitar los procesos de aprendizaje que acompañen la evolución y el desarrollo de los niños. Esta sería, a nuestro juicio, la única forma de garantizar la universalización de la educación para las personas con discapacidad. Este sería un componente fundamental para la disminución de la vulnerabilidad social y de la exclusión que, muchas veces, se generan cuando los niños y niñas transitan por sistemas educativos segregados. La posibilidad de acceder a la educación superior debería ser una elección, mientras que no acceder no debería ser una determinación por falta de oportunidades. Incluir los temas de discapacidad en la agenda de investigación en los ámbitos académicos es otro componente esencial para avanzar en la perspectiva de derechos.

\section{REFERENCIAS}

ESPAÑA. Ministerio de Educación y Ciencia. Declaración de Salamanca y Marco de Acción para las Necesidades Educativas Especiales. Salamanca: UNESCO, 1994.

GÓMEZ-BELEÑO, G.; LÓPEZ-MUÑOZ, Y. Tecnología de asistencia para la inclusión educativa de personas con parálisis cerebral: una revisión crítica de la literatura. Rehabilitación, SERMEF, 50 (2), p. 87-94, 2016. 
LUQUE-PARRA, D.; RODRÍGUEZ-INFANTE, G. Y.; LUQUE-ROJAS, M. Adecuación del curriculum al alumnado universitario con discapacidad: un estudio de caso. Revista Iberoamericana de Educación Superior, v. 5, n. 13, p. 101-116, 2016.

NACIONES UNIDAS (ONU). Convención sobre los Derechos de las Personas con Discapacidad (CDPD). 2006.

ORGANIZACIÓN MUNDIAL DE LA SALUD (OMS). Clasificación del Funcionamiento, de la Discapacidad y de la Salud (CIF). Versión en español. IMSERSO. España, 2011a. ORGANIZACIÓN MUNDIAL DE LA SALUD (OMS). Informe Mundial sobre la Discapacidad. Banco Mundial, 2011b.

TENORIO EITEL, S.; RAMÍREZ-BURGOS, M. Experiencia de inclusión en educación superior de estudiantes en situación de discapacidad sensorial. Educ. Educ., v. 19, n. 1, p. 9-28, 2016.

UNIVERSIDAD DE LA REPÚBLICA (UDELAR). CENSO DE ESTUDIANTES DE GRADO DE LA UNIVERSIDAD DE LA REPÚBLICA, 7, 2012. Uruguay. Anais... Uruguay: UdelaR, 2012. Recuperado de http://www.universidad.edu.uy/renderPage/ index/pageId/129. Acceso en: fev. 2017.

YUPANQUI CONCHA, A.; ARANDA FARÍAS, C.; VAZQUEZ OYARZUN, C.; VERDUGO HUENUMÁN, W. Educación Inclusiva y discapacidad: su incorporación en la formación profesional de la educación superior. Revista de Educación Superior, v. XLIII (3), n. 171, p. 93-115, 2014.

Texto recibido el 05 de marzo de 2017. Texto aprobado el 25 de marzo de 2017. 